\title{
Sur les caractéristiques chimiques du sous-écoulement d'un ruisseau des Pyrénées
}

\author{
R. Rouch1 \\ M. Bakalowiczl \\ A. Mangin! \\ D. D'Hulst ${ }^{1}$
}

Mots clés : milieu interstitiel hyporhéique, hydrochimie.

Le chimisme du sous-écoulement du tuisseau de Lachein (Pyrénées) a été étudié sur un site de $75 \mathrm{~m}^{2}$, à partir de prélèvements effectués à $60 \mathrm{~cm}$ de profondeur, pendant la durée d'un cycle hydrologique.

Les eaux du sous-écoulement et celles du cours de surface ont des caractéristiques chimiques proches qui évoluent de façon synchrone au fil des saisons. Toutefois, cette analogie n'implique pas une liaison directe entre ces deux types d'eau. L'étude du système calco-carbonique montre, en effet, que les eaux de surface et celles du sous-écoulement évoluent de façon indépendante et que leurs échanges sont limités.

Sur le site étudié, la chimie des eaux du sous-écoulement est caractérisée par son hétérogénéité spatiale. Cette hétérogénéité est liée aux différents ensembles physiographiques définis par la dynamique des écoulements de surface.

Un modèle de fonctionnement est proposé rendant compte des relations existant aux différents niveaux d'échelle.

On the chemical characteristics of a Pyrenean brook underflow.

Keywords : hyporheic habitat, hydrochemistry.

The water chemistry of the ruisseau de Lachein underflow (Pyrenees) was studied on a $75 \mathrm{~m}^{2}$ area, at a $60 \mathrm{~cm}$ depth and during a hydrological cycle.

The underflow and surface waters have closed chemical characteristics which evolve synchronously throughout the year. Yet, this analogy does not involve a direct relation between these two waters, for the calco-carbonic parameters show that underflow and surface waters evolve independently with limited exchanges.

In the study area, the underflow chemistry is characterized by its spatial heterogeneity. This heterogeneity is connected to the different physiographic units defined by the surface flows.

A functional model is proposed showing the existing relationships at different scale levels.

Dans le sous-écoulement du ruisseau de Lachein (fig. 1), affluent du Lez (Ariège), l'étude de la microrépartition des Crustacés sur une aire de $75 \mathrm{~m}^{2} \mathrm{mon}$ tre que les formes stygobies de ce groupe ne sont pas distribuées de façon homogène (Rouch 1988). La répartition de chacune d'entre elles peut être définie à partir des ensembles physiographiques déterminés par les écoulements de surface (fig. 2): une

1. Laboratoire souterrain du CNRS, Moulis, 09200 Saint-Girons, France. zone de creusement (chenal), une zone d'eau morte et deux zones de dépôts. Chacun de ces ensembles est caractérisé par des valeurs différentes de la granulométrie, de la porosité et de la teneur en oxygène dissous (Rouch ibid.).

Sur le plan chimique, les eaux du sous-écoulement de ce ruisseau présentent, en moyenne, des caractéristiques toujours voisines de celles du cours subaérien (Rouch ibid.).

Le but du présent travail a consisté à rechercher si, parallèlement à l'organisation du peuplement 


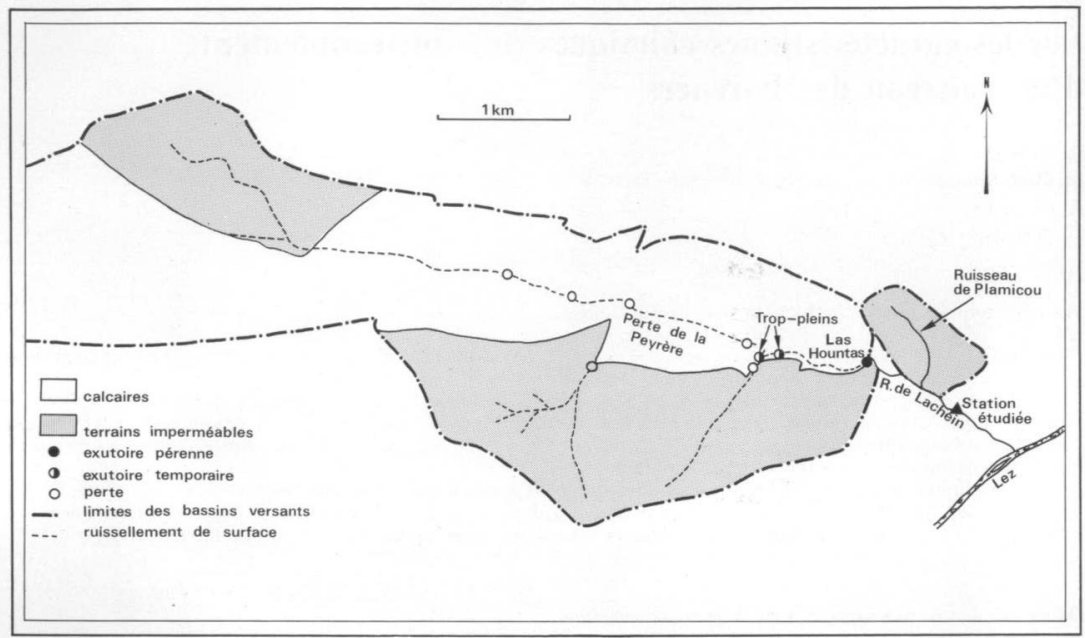

Fig. 1. Bassin versant du ruisseau de Lachein. L'essentiel de l'alimentation de ce ruisseau provient du systeme karstique du Bəget dont seul l'aval a été représenté.

mise en évidence pour les Crustacés, il existait, dans le secteur étudié, une structure de la chimie des eaux et quelles en étaient les causes éventuelles.

\section{Le site étudié, méthodes, paramètres analysés}

Le ruisseau de Lachein (fig, 1) est, pour l'essentiel, alimenté par le bassin versant $\left(13,25 \mathrm{~km}^{2}\right) \mathrm{du}$ système karstique dı Baget. En effet, il prend sa source à Las Hountas, exutoire pérenne de ce système. $500 \mathrm{~m}$ en aval de cet te source, il reçoit un affluent en rive gauche, le ruisseau de Plamicou, dont le bassin versant ne représente que $1 \mathrm{~km}^{2}$.

En raison de leur origine, les eaux de surface du ruisseau de Lachein présentent, globalement, les caractéristiques typiques d'un bassin versant calcaire (Rouch 1988) avec un fort pourcentage de bicarbonates (70 à $89 \%$ des anions) et de calcium (plus de $80 \%$ des cations). Leur conductivité est comprise entre 250 et $315 \mu \mathrm{S} / \mathrm{cm}$. Les teneurs en sulfates, plus élevées que la moyenne des bassins karstiques (14 à $53 \mathrm{mg} / \mathrm{l}$ ), sont dues au ruisseau de Plamicou qui draine des argiles et des marnes gypsifères attribuées au Trias.

L'aire étudiée correspond à une portion du ruisseau de Lachein située $830 \mathrm{~m}$ en aval de la source. Les $75 \mathrm{~m}^{2}$ du site ont été divisés en 10 secteurs désignés par les let tres A à J (fig. 3). Tous les deux mois, de septembre 1985 à juillet 1986, vingt pompages (Bou \& Rouch 1967), soit deux par secteur, ont été réalisés au moyen d'une sonde enfoncée à $60 \mathrm{~cm}$ de profondeur dans les sédiments. Une série de contrôle de 10 prélèvements a été effectuée en septembre 1986. Rappelons que, afin d'éviter les problèmes posés par des sondes fixées à demeure, chacun de ces prélèvements correspond à un emplacement unique, repéré par ses coordonnées par rapport au quadrillage pré-établi. Chaque échantillon a été identifié par la lettre du secteur dont il était issu et par 


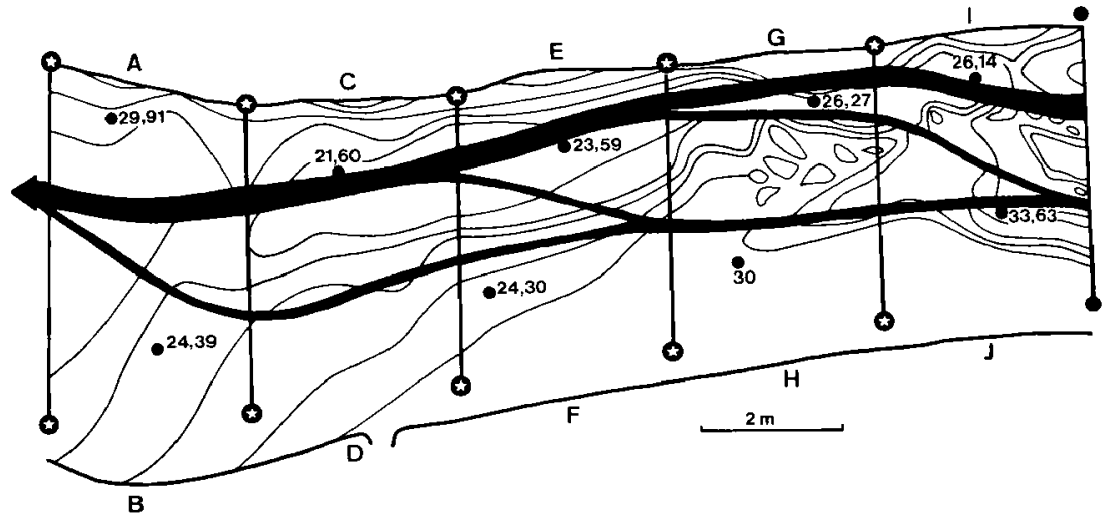

Fig. 2. Carte du site étudié. Les lignes de niveau sont figurées en traits fins, les écoulements principaux en traits épais. Les valeurs de la porosité (pourcentage) sont indiquées pour neuf points du site.

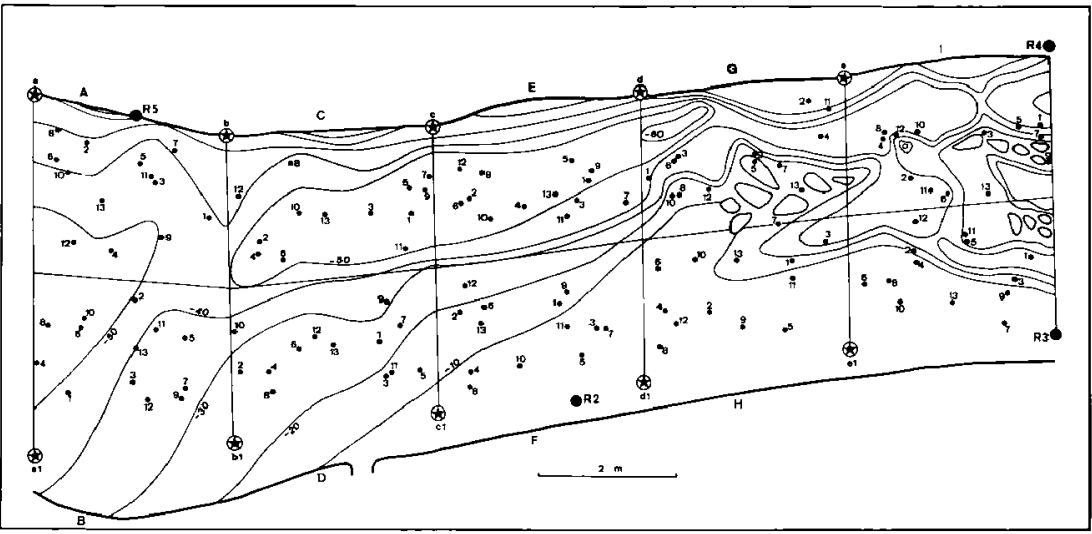

Fig. 3. Position des échantillons dans les différents secteurs (A à J) du site étudié. 


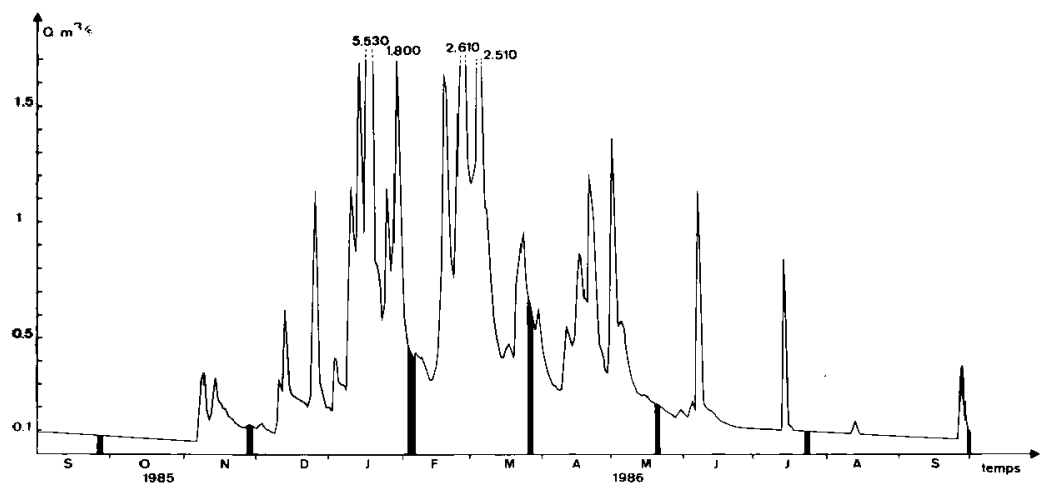

Fig. 4. Débits du ruisseau de Lachein et périodes de prélèvements

un numéro: 1.2 lors de l'échantillonnage de septembre $1985,3-4$ pour celui de novembre $1985, \ldots 13$ pour celu i de septembre 1986. Ces sept séries de prélèvements correspondent à des basses eaux (septembre et novembre 1985, juillet et septembre 1986) et à des hautes eaux (février, mars, mai 1986)(fig. 4).

Dans chaque prélèvement, $250 \mathrm{~cm}^{3}$ étaient destinés aux analyses chimiques. Celles-ci ont été effectuées au laboratoire selon les procédures classiques (Bakalowicz 1979). 10 variables ont été mesurées : le $\mathrm{pH}$ et les teneurs totales en calcium, magnésium, sodium, potassium, silice, nitrate, chlorure, sulfate et bicarbonate, à partir desquelles ont été calculés les équilibres chimiques qui ont permis d'extraire deux variables supplémentaires, la pression partielle en $\mathrm{CO}_{2}\left(\mathrm{pCO}_{2}\right)$ et l'indice de saturation par rapport à la calcite $(\mathrm{dpH})$. Le tableau des données comprend donc 137 prélèvements (dont 7 pour le cours d'eau superficiel) et 12 variables chimiques ${ }^{1}$.

\section{Caractéristiques générales}

Il est habituel d'interpréter les variations du chimisme des eaux en terme de mélange entre un pôle

1. Du fait de ses dimensions, le tableau des donnees n'est pas reproduit dans cette note. Il peut être fourni sur demande. concentré, résidant dans le milieu, et un pôle moins concentré représenté par l'eau de pluie ou par une eau transitant rapidement. Or, les réactions chimiques de mise en solution nécessitent un temps plus ou moins long pour atteindre l'équilibre. Les concentrations dans la solution peuvent, par conséquent, évoluer sous l'effet de la variation du temps de séjour dans un milieu donné. Si ce milieu est hétéregène, les temps de séjour seront variables dans l'espace, ce qui entraînera une variabilité chimique. đIl est donc nécessaire de prendre en compte à la fois le 'mélange d'eaux de qualités chimiques différentes et leur temps de séjour pour interpréter les variations du chimisme.

Dans le ruisseau de Lachein, l'essentiel du chimisme est lié à la dissolution des carbonates. En effet, les variables du système calco-carbonique (Ca, $\mathrm{Mg}, \mathrm{HCO}_{3}, \mathrm{dpH}$ et $\mathrm{pCO}_{2}$ ) représentent plus de $90 \%$ de l'information chimique globale.

Le système calco-carbonique est un système hétérogène dans lequel interviennent trois phases: la phase gazeuse $\left(\mathrm{CO}_{2}\right)$, la solution et la phase solide (carbonate de calcium et de magnésium). Toute variation de la $\mathrm{pCO}_{2}$ du gaz induit une variation correspondante de celle de la solution et, donc, du pH, du $\mathrm{Ca}$ et du $\mathrm{HCO}_{3}$. Si le temps de réaction est suffisant, les équilibres sont atteints et les teneurs en $\mathrm{Ca}$ et $\mathrm{HCO}_{3}$, de même que le $\mathrm{pH}$, sont totalement 
fixées par la $\mathrm{pCO}_{2}$. Mais, en général, les équilibres ne sont pas complètement réalisés ; par conséquent, le $\mathrm{pH}$ et les teneurs en $\mathrm{Ca}$ et $\mathrm{HCO}_{3}$ sont fonction a la fois de la $\mathrm{pCO}_{2}$ et de l'écart entre l'état effectif de la solution (mesuré par le $\mathrm{pH}$ réel) et l'état théorique d'équilibre calculé (fourni par le $\mathrm{pH}$ d'équilibre); cet écart est donné par la variable $\mathrm{dpH}$, à condition que le $\mathrm{pH}$ corresponde uniquement au système calco-carbonique. L'interprétation et la recherche des causes de variation de ce système ne peuvent donc porter que sur les deux variables $\mathrm{PCO}_{2}$ et dpH et non, comme cela est souvent pratiqué, sur le $\mathrm{pH}, \mathrm{Ca}$ et $\mathrm{HCO}_{3}$.

Les autres variables chimiques, externes au système calco-carbonique, résultent toutes de la mise en solution de solides sans contribution d'une phase gazeuse. Leurs variations sont par conséquent beaucoup plus simples à interpréter, d'autant plus qu'elles sont toutes observées à des teneurs très faibles, dans le domaine des fortes sous-saturations. Elles constituent cependant une information complementaire intéressante.

\section{Description sommaire des données}

Le tableau I résume les données hydrochimiques du sous-écoulement et du ruisseau en fonction du temps. Toutes les variables sont soumises à une évolution saisonnière marquée. Les valeurs maximales observées apparaissent à des époques différentes mais de façon concomitante pour le ruisseau et le sous-écoulement : en septembre 1985 pour $\mathbf{C a}, \mathbf{M g}$. $\mathrm{Na}, \mathrm{SO}_{4}, \mathrm{pH}$ et dpH ; en février 1986 pour $\mathrm{pCO}_{2}$ et $\mathrm{HCO}_{3}$; en septembre 1986 pour $\mathrm{SiO}_{2}$ et $\mathrm{NO}_{3}$. Ainsi, ces maximums sont observés soit lors de l'étiage, soit lors des premières crues du cycle comme cela a été mis en évidence à l'exu toire du système kars. tique du Baget (Bakalowicz \& Aminot 1974).

Les minimums apparaissent en général pendant la période de hautes eaux, jusqu'au début de l'étiage (février à juillet 1986), cornme c'est également le cas au Baget. Mais ces minimums, dispersés selon les variables, ne coïncident pas toujours dans le ruisseau et le sous-écoulement, notamment pour le $\mathrm{pH}$, le magnésium, le potassium, les nitrates et la $\mathrm{pCO}_{2}$. Ceci implique des relations complexes entre ces deux types d'eau.

L'eau du sous-écoulement montre, en moyenne, par rapport au ruisseau, une différence très significative en ce qui concerne le système calcocarbonique $\left(\mathrm{pH}, \mathrm{HCO}_{3}, \mathrm{dpH}, \mathrm{pCO}_{2}\right.$ et, pendant les hautes eaux, Ca). Depuis la source de Las Hountas, les eaux superficielles sont soumises à une

\begin{tabular}{|c|c|c|c|c|c|c|c|c|c|c|c|c|c|c|}
\hline datas & & & pH & $\mathrm{Ca}$ & $\frac{\mathrm{Mg}}{\mathrm{mg} / \mathrm{l}}$ & $\underset{\mathrm{m} \mathscr{\mathrm { Na }} / \mathrm{I}}{\mathrm{Na}}$ & $\underset{m g / 1}{R}$ & $\mid \begin{array}{l}\mathrm{SiO} 2 \\
\mathrm{mg} / 1\end{array}$ & $\begin{array}{r}\mathbf{N O 3} \\
\mathrm{m} \& / 1\end{array}$ & $\begin{array}{c}\mathrm{Cl} \\
\mathrm{mg} / 1\end{array}$ & $\mid \begin{array}{r}\mathrm{SO}_{4} \\
\mathrm{xg} / 1\end{array}$ & $\begin{array}{l}5003 \\
\mathrm{mo} / 1\end{array}$ & $5 \mathrm{phl}$ & $\begin{array}{c}\mathrm{pOO}_{2} \\
\%\end{array}$ \\
\hline Sept 85 & $\begin{array}{l}\mathrm{E} \\
\mathrm{S}\end{array}$ & $\begin{array}{l}\mathbf{x} \\
\sigma\end{array}$ & $\begin{array}{l}8.30 \\
7.86 \\
0.15\end{array}$ & $\begin{array}{l}66.1 \\
66.7 \\
1.89\end{array}$ & $\begin{array}{l}6.60 \\
6.25 \\
0.31\end{array}$ & $\begin{array}{l}1.50 \\
1.45 \\
0.10\end{array}$ & $\begin{array}{l}0.70 \\
0.61 \\
0.07\end{array}$ & $\begin{array}{l}2.7 \\
2.2 \\
1.45\end{array}$ & \begin{tabular}{ll|}
1.19 \\
0.05 \\
0.07
\end{tabular} & $\begin{array}{l}2.05 \\
1.75 \\
0.07\end{array}$ & $\begin{array}{l}53.5 \\
46.5 \\
2.47\end{array}$ & $\mid \begin{array}{r}164.7 \\
171.6 \\
7.05\end{array}$ & $\left\{\begin{array}{l}0.68 \\
0.25 \\
0.12\end{array}\right.$ & $\left\{\begin{array}{l}0.07 \\
0.21 \\
0.11\end{array}\right.$ \\
\hline Nov 85 & $\begin{array}{l}\mathrm{B} \\
\mathrm{S}\end{array}$ & $\begin{array}{l}x \\
0\end{array}$ & $\begin{array}{l}8.16 \\
7.81 \\
0.14\end{array}$ & $\begin{array}{l}63.5 \\
63.9 \\
1.13\end{array}$ & $\begin{array}{l}6.20 \\
6.15 \\
0.27\end{array}$ & $\begin{array}{l}1.25 \\
1.20 \\
0.04\end{array}$ & $\begin{array}{l}0.56 \\
0.70 \\
0.10\end{array}$ & $\begin{array}{l}2.4 \\
2.5 \\
1.08\end{array}$ & $\begin{array}{l}1.94 \\
0.69 \\
0.62\end{array}$ & $\begin{array}{l}2.20 \\
2.25 \\
0.11\end{array}$ & $\mid \begin{array}{l}37.0 \\
36.0 \\
2.19\end{array}$ & $\begin{array}{r}170.8 \\
176.9 \\
6.34\end{array}$ & $\begin{array}{l}0.53 \\
0.20 \\
0.13\end{array}$ & $\mid \begin{array}{l}0.09 \\
0.24 \\
0.10\end{array}$ \\
\hline Dor 86 & $\begin{array}{l}8 \\
5\end{array}$ & $\begin{array}{l}x \\
a\end{array}$ & $\begin{array}{l}8.21 \\
7.69 \\
0.07\end{array}$ & $\begin{array}{l}60.8 \\
62.4 \\
1.26\end{array}$ & $\begin{array}{l}4.70 \\
4.50 \\
0.39\end{array}$ & $\begin{array}{l}1.20 \\
1.40 \\
0.58\end{array}$ & $\begin{array}{l}0.74 \\
0.46 \\
0.12\end{array}$ & $\left\{\begin{array}{l}1.7 \\
2.2 \\
1.10\end{array}\right.$ & $\begin{array}{l}0.03 \\
0.17 \\
0.24\end{array}$ & $\begin{array}{l}2.10 \\
2.10 \\
0.07\end{array}$ & $\begin{array}{l}21.0 \\
20.0 \\
2.17\end{array}$ & $\begin{array}{r}176.3 \\
183.5 \\
3.66\end{array}$ & $\begin{array}{l}0.57 \\
0.10 \\
0.07\end{array}$ & $\begin{array}{l}0.09 \\
0.31 \\
0.05\end{array}$ \\
\hline Mars 86 & $\begin{array}{l}R \\
S\end{array}$ & $\begin{array}{l}x \\
\sigma\end{array}$ & $\begin{array}{l}8.10 \\
7.70 \\
0.15\end{array}$ & $\mid \begin{array}{l}54.9 \\
56.8 \\
2.03\end{array}$ & $\begin{array}{l}4.60 \\
4.45 \\
0.14\end{array}$ & $\begin{array}{l}1.10 \\
1.30 \\
0.15\end{array}$ & $\begin{array}{l}0.53 \\
0.47 \\
0.11\end{array}$ & $\begin{array}{l}2.6 \\
3.3 \\
0.45\end{array}$ & $\begin{array}{l}0.78 \\
2.08 \\
0.81\end{array}$ & $\begin{array}{l}1.55 \\
1.70 \\
0.07\end{array}$ & $\begin{array}{l}13.7 \\
14.9 \\
1.38\end{array}$ & $\begin{array}{r}162.3 \\
168.7 \\
8.47\end{array}$ & $\begin{array}{l}0.39 \\
0.04 \\
0.12\end{array}$ & $\begin{array}{l}0.08 \\
0.30 \\
0.15\end{array}$ \\
\hline MaL 86 & $\begin{array}{l}\mathrm{R} \\
\mathrm{S}\end{array}$ & $\sigma$ & $\begin{array}{l}8.22 \\
7.83 \\
0.11\end{array}$ & $\begin{array}{l}57.6 \\
58.7 \\
2.85\end{array}$ & $\begin{array}{l}4.20 \\
4.65 \\
0.91\end{array}$ & $\begin{array}{l}1.30 \\
1.40 \\
0.02\end{array}$ & $\begin{array}{l}0.40 \\
0.51 \\
0.69\end{array}$ & $\begin{array}{l}3.2 \\
3.3 \\
0.42\end{array}$ & $\begin{array}{l}1.00 \\
1.25 \\
0.33\end{array}$ & $\begin{array}{l}1.50 \\
1.50 \\
0.11\end{array}$ & $\begin{array}{l}17.0 \\
14.0 \\
1.44\end{array}$ & $\begin{array}{r}161 \\
169.2 \\
6.32\end{array}$ & $\begin{array}{l}0.53 \\
0.19 \\
0.09\end{array}$ & $\begin{array}{l}0.08 \\
0.21 \\
0.07\end{array}$ \\
\hline Jut1 86 & $\begin{array}{l}R \\
S\end{array}$ & $\begin{array}{l}x \\
\sigma\end{array}$ & $\begin{array}{l}8.15 \\
7.77 \\
0.15\end{array}$ & $\begin{array}{l}57.7 \\
59.0 \\
1.52\end{array}$ & $\begin{array}{l}4.90 \\
4.80 \\
0.17\end{array}$ & $\begin{array}{l}1.02 \\
1.00 \\
0.02\end{array}$ & $\begin{array}{l}0.42 \\
0.51 \\
0.10\end{array}$ & $\begin{array}{l}3.0 \\
3.5 \\
0.27\end{array}$ & $\begin{array}{l}1.95 \\
2.07 \\
0.61\end{array}$ & $\begin{array}{l}1.50 \\
1.45 \\
0.07\end{array}$ & $\begin{array}{l}24.0 \\
23.5 \\
2.37\end{array}$ & $\begin{array}{r}159.8 \\
163.2 \\
6.55\end{array}$ & $\begin{array}{l}0.48 \\
0.11 \\
0.13\end{array}$ & $\begin{array}{l}0.09 \\
0.25 \\
0.12\end{array}$ \\
\hline Sept. 86 & $\begin{array}{l}R \\
S\end{array}$ & $\begin{array}{l}\mathbf{x} \\
\sigma\end{array}$ & $\begin{array}{l}8.20 \\
7.78 \\
0.12\end{array}$ & $\begin{array}{l}62.1 \\
63.7 \\
1.60\end{array}$ & $\begin{array}{l}5.60 \\
5.60 \\
0.16\end{array}$ & \begin{tabular}{|l|}
1.12 \\
1.10 \\
0.04
\end{tabular} & $\begin{array}{l}0.62 \\
0.60 \\
0.06\end{array}$ & \begin{tabular}{|l|}
3.6 \\
4.0 \\
0.28
\end{tabular} & \begin{tabular}{|l|}
4.80 \\
2.63 \\
0.52
\end{tabular} & $\begin{array}{l}1.70 \\
1.95 \\
0.36\end{array}$ & $\begin{array}{l}37.0 \\
37.0 \\
0.93\end{array}$ & $\begin{array}{r}157.4 \\
162.7 \\
4.92\end{array}$ & $\begin{array}{l}0.55 \\
0.14 \\
0.10\end{array}$ & $\begin{array}{l}0.08 \\
0.23 \\
0.08\end{array}$ \\
\hline
\end{tabular}

Tableau I. Caractéristiques chimiques du ruisseau de Lachein $(R)$ et de son sous-écuulement $(S) ; x=$ valeur moyenne; $\sigma=$ écart-type. 
évolution chimique due au départ du $\mathrm{CO}_{2}$ dans l'atmosphère : diminution de la $\mathrm{pCO}_{2}$, de $\mathrm{HCO}_{3}$ et $\mathrm{Ca}$, augmentation corrélative de $\mathrm{pH}$ et de $\mathrm{dpH}$. Les eaux du sous-écoulement conservent, au contraire, les caractères très nets d'eaux souterraines : ceci signifie que le sous-écoulement ne peut pas être considéré comme étant directement alímenté par le ruisseau, au moins à proximité du site d'étude. D'autres éléments soulignent cette différence. Les teneurs en sodium et en silice sont plus élevées dans le sousécoulement, alors que les teneurs en sulfates y sont plus faibles. Nitrates et potassium montrent toujours des différences nettes, positives ou négatives. entre le ruisseau et le sous-écoulement.

Le tableau II rassemble les caractéristiques moyennes de chacun des secteurs au cours de la période étudiće. L'hétérogénéité du site est évidente. Certains secteurs (B, notamment) se différencient très nettement du ruisseau alors que d'autres s'en distinguent moins, comme le secteur $\mathbf{J}$. Toutefois, il existe une grande similitude du système calcocarbonique des différentes secteurs qui s'opposent au ruisseau par leur $\mathrm{pCO}_{2}$ nettement plus forte et leur situation nettement plus proche de l'équilibre chimique.

\section{Analyse multidimensionnelle des données}

Etant donné le nombre élevé de variables et d'échantillons, l'analyse multidimensionnelle du tableau de données s'imposait afin de mieux étudier les structures des variations observées. Pui sque l'ensemble de ces données présente une structure temporelle et spatiale, le temps et l'espace ont été considérés comme des variables qualitatives implicites qui pouvaient être ajoutées aux variables quantitatives du tableau initial. Il a été fait appel à l'analyse factorielle discriminante (AFD) qui permet de prendre en compte de telles variables qualitatives.

\subsection{Evolution du chimisme en fonction du temps}

La variable qualitative temps a été introduite selon les modalités suivantes : 1 pour les échantillons de septembre 1985, 2 pour ceux de novembre

\begin{tabular}{|c|c|c|c|c|c|c|c|c|c|c|c|c|c|}
\hline secteur & & $\mathrm{pH}$ & $\begin{array}{c}\mathrm{Ca} \\
\mathrm{mg} / 1\end{array}$ & $\begin{array}{c}\mathrm{Mg} \\
\operatorname{mg} / 1\end{array}$ & $\mathrm{Na}$ & $\begin{array}{c}K \\
3198 / 1\end{array}$ & $\mid \begin{array}{l}\mathrm{SiO}_{2} \\
\mathrm{mg} / 1\end{array}$ & $\begin{array}{r}\mathrm{NO}_{3} \\
\mathrm{mg} / 1\end{array}$ & $\begin{array}{c}\mathrm{Cl} \\
\mathrm{mg} / 1\end{array}$ & $\begin{array}{r}\mathrm{SO}_{4} \\
\mathrm{me} / 1\end{array}$ & $\begin{array}{l}\mathrm{HCO}_{3} \\
\mathrm{me} / 1\end{array}$ & $8 \mathrm{pH}$ & $\underset{\%}{\mathrm{PCO}_{2}}$ \\
\hline A & $\begin{array}{l}x \\
\sigma\end{array}$ & $\begin{array}{l}7.83 \\
0.15\end{array}$ & $\mid \begin{array}{r}61.1 \\
3.3\end{array}$ & $\begin{array}{l}5.1 \\
0.9\end{array}$ & $\left\{\begin{array}{l}1.2 \\
0.2\end{array}\right.$ & $\begin{array}{l}0.53 \\
0.09\end{array}$ & $\begin{array}{l}2.5 \\
1.5\end{array}$ & $\begin{array}{l}1.2 \\
1.2\end{array}$ & $\begin{array}{l}1.8 \\
0.2\end{array}$ & $\begin{array}{l}27 \\
12\end{array}$ & $\begin{array}{r}168 . \\
9\end{array}$ & $\begin{array}{l}0.19 \\
0.15\end{array} \mid$ & $\begin{array}{l}0.22 \\
0.08\end{array}$ \\
\hline B & $\begin{array}{l}x \\
\sigma\end{array}$ & $\begin{array}{l}7.58 \\
0.12\end{array}$ & $\begin{array}{r}64.0 \\
3.7\end{array}$ & $\begin{array}{l}5.0 \\
0.7\end{array}$ & $\begin{array}{l}1.2 \\
0.2\end{array}$ & $\left\{\begin{array}{l}0.71 \\
0.07\end{array}\right.$ & $\begin{array}{l}3.3 \\
1.0\end{array}$ & $\begin{array}{l}1.0 \\
0.8\end{array}$ & $\begin{array}{l}1.8 \\
0.2\end{array}$ & $\begin{array}{l}24 \\
11\end{array}$ & $\begin{array}{r}183 \\
8\end{array}$ & $\begin{array}{l}0.02 \\
0.12\end{array}$ & $\begin{array}{l}0.41 \\
0.12\end{array}$ \\
\hline C & $\begin{array}{l}\mathbf{x} \\
\boldsymbol{\sigma}\end{array}$ & $\begin{array}{l}7.79 \\
0.12\end{array}$ & $\begin{array}{r}61.1 \\
3.6\end{array}$ & $\begin{array}{l}5.1 \\
0.9\end{array}$ & $\begin{array}{l}1.2 \\
0.2\end{array}$ & $\begin{array}{l}0.52 \\
0.10\end{array}$ & $\begin{array}{l}2.9 \\
0.8\end{array}$ & $\begin{array}{l}1.1 \\
0.9\end{array}$ & $\begin{array}{l}1.9 \\
0.4\end{array}$ & $\begin{array}{l}27 \\
11\end{array}$ & $\begin{array}{r}171 \\
8\end{array}$ & $\begin{array}{l}0.16 \\
0.12\end{array}$ & $\begin{array}{l}0.24 \\
0.07\end{array}$ \\
\hline D & $\begin{array}{l}x \\
\sigma\end{array}$ & $\left\{\begin{array}{l}7.70 \\
0.16\end{array}\right.$ & $\begin{array}{r}62.9 \\
3.8\end{array}$ & $\begin{array}{l}5.2 \\
0.8\end{array}$ & $\begin{array}{l}1.2 \\
0.1\end{array}$ & $\begin{array}{l}0.61 \\
0.08\end{array}$ & $\begin{array}{l}3.1 \\
0.7\end{array}$ & $\begin{array}{l}1.2 \\
1.2\end{array}$ & $\begin{array}{l}1.8 \\
0.3\end{array}$ & $\begin{array}{l}26 \\
12\end{array}$ & $\begin{array}{r}178 \\
11\end{array}$ & $\begin{array}{l}0.10 \\
0.13\end{array}$ & $\begin{array}{l}0.32 \\
0.16\end{array}$ \\
\hline$E$ & $\begin{array}{l}\mathbf{x} \\
\sigma\end{array}$ & $\begin{array}{l}7.76 \\
0.11\end{array}$ & $\begin{array}{r}61.3 \\
3.4\end{array}$ & $\begin{array}{l}5.3 \\
0.7\end{array}$ & $\begin{array}{l}1.4 \\
0.7\end{array}$ & $\begin{array}{l}0.54 \\
0.08\end{array}$ & $\begin{array}{l}3.8 \\
0.7\end{array}$ & $\begin{array}{l}1.2 \\
1.0\end{array}$ & $\begin{array}{l}1.8 \\
0.3\end{array}$ & $\begin{array}{l}27 \\
11\end{array}$ & $\begin{array}{r}171 \\
7\end{array}$ & $\left.\begin{array}{l}0.13 \\
0.11\end{array}\right]$ & $\begin{array}{l}0.25 \\
0.06\end{array}$ \\
\hline$F$ & $\begin{array}{l}x \\
\sigma\end{array}$ & $\begin{array}{l}7.83 \\
0.08\end{array}$ & $\mid \begin{array}{r}61.2 \\
3.6\end{array}$ & $\begin{array}{l}5.3 \\
0.9\end{array}$ & $\begin{array}{l}1.2 \\
0.1\end{array}$ & $\begin{array}{l}0.59 \\
0.08\end{array}$ & $\begin{array}{l}3.1 \\
0.8\end{array}$ & $\begin{array}{l}1.4 \\
0.9\end{array}$ & {$\left[\begin{array}{l}1.8 \\
0.3\end{array}\right.$} & $\begin{array}{l}26 \\
12\end{array}$ & $\begin{array}{r}170 \\
8\end{array}$ & $\begin{array}{l}0.20 \\
0.08\end{array}$ & $\left\{\begin{array}{l}0.21 \\
0.04\end{array}\right.$ \\
\hline G & $\begin{array}{l}\mathbf{x} \\
\sigma\end{array}$ & $\begin{array}{l}7.74 \\
0.09\end{array}$ & $\begin{array}{r}61.6 \\
3.4\end{array}$ & $\begin{array}{l}5.1 \\
0.6\end{array}$ & $\begin{array}{l}1.3 \\
0.1\end{array}$ & $\begin{array}{l}0.45 \\
0.12\end{array}$ & $\begin{array}{l}3.2 \\
0.8\end{array}$ & $\begin{array}{l}1.0 \\
0.9\end{array}$ & $\begin{array}{l}1.8 \\
0.3\end{array}$ & $\begin{array}{l}26 \\
12\end{array}$ & $\begin{array}{r}171 \\
6\end{array}$ & $\mid \begin{array}{l}0.11 \\
0.10\end{array}$ & $\left\{\begin{array}{l}0.27 \\
0.06\end{array}\right.$ \\
\hline $\mathrm{H}$ & $\begin{array}{l}\mathbf{x} \\
\sigma\end{array}$ & $\begin{array}{l}7.86 \\
0.07\end{array}$ & $\begin{array}{r}60.5 \\
3.3\end{array}$ & $\begin{array}{l}5.1 \\
0.8\end{array}$ & 1.3 & $\begin{array}{l}0.55 \\
0.10\end{array}$ & $\begin{array}{l}2.2 \\
1.2\end{array}$ & $\begin{array}{l}1.2 \\
1.2\end{array}$ & $\begin{array}{l}1.8 \\
0.3\end{array}$ & $\begin{array}{l}27 \\
12\end{array}$ & $\begin{array}{r}168 \\
8\end{array}$ & $\left(\begin{array}{l}0.22 \\
0.08\end{array}\right.$ & $\begin{array}{l}0.19 \\
0.04\end{array}$ \\
\hline I & $\begin{array}{l}\mathbf{x} \\
\sigma\end{array}$ & $\left\{\begin{array}{l}7.78 \\
0.13\end{array}\right.$ & $\mid \begin{array}{r}60.3 \\
4.0\end{array}$ & $\begin{array}{l}5.3 \\
1.2\end{array}$ & $\begin{array}{l}1.3 \\
0.2\end{array}$ & $\begin{array}{l}0.45 \\
0.15\end{array}$ & 2.5 & $\begin{array}{l}1.1 \\
1.0\end{array}$ & $\begin{array}{l}1.7 \\
0.3\end{array}$ & $\begin{array}{l}27 \\
12\end{array}$ & $\begin{array}{r}168 \\
7\end{array}$ & {$\left[\begin{array}{l}0.14 \\
0.13\end{array}\right.$} & $\left\{\begin{array}{l}0.24 \\
0.68\end{array}\right.$ \\
\hline $\mathrm{J}$ & $\begin{array}{l}\mathbf{x} \\
\sigma\end{array}$ & $\begin{array}{l}7.88 \\
0.13\end{array}$ & $\begin{array}{r}60.3 \\
3.3\end{array}$ & $\begin{array}{l}5.0 \\
0.9\end{array}$ & $\left\{\begin{array}{l}1.3 \\
0.2\end{array}\right.$ & $\begin{array}{l}0.51 \\
0.13\end{array}$ & $\begin{array}{l}2.6 \\
0.7\end{array}$ & $\begin{array}{l}1.2 \\
1.1\end{array}$ & {$\left[\begin{array}{l}1.8 \\
0.3\end{array}\right.$} & $\begin{array}{l}26 \\
12\end{array}$ & $\begin{array}{r}167 \\
7\end{array}$ & $\begin{array}{l}0.23 \\
0.14\end{array}$ & $\left\{\begin{array}{l}0.19 \\
0.06\end{array}\right.$ \\
\hline ruteseau & $\begin{array}{l}x \\
0\end{array}$ & $\begin{array}{l}8.19 \\
0.06\end{array}$ & $\begin{array}{r}60.4 \\
3.6\end{array}$ & $\begin{array}{l}5.3 \\
0.8\end{array}$ & $\begin{array}{l}1.2 \\
0.1\end{array}$ & $\begin{array}{l}0.57 \\
0.12\end{array}$ & $\begin{array}{l}2.7 \\
0.6\end{array}$ & $\begin{array}{l}1.7 \\
1.4\end{array}$ & $\begin{array}{l}1.8 \\
0.3\end{array}$ & $\begin{array}{l}29 \\
13\end{array}$ & $\begin{array}{r}165 \\
6\end{array}$ & $\mid \begin{array}{l}0.53 \\
0.08\end{array}$ & $\begin{array}{l}0.09 \\
0.01\end{array}$ \\
\hline
\end{tabular}

Tableau 2. Valeurs moyennes et écarts-lypes des caractèristiques chimiques du ruisseau de Lachein et du sous-écoulement des différents secteurs étudiés. 
1985... 7 pour ceux de septembre 1986. Les échantillons du ruisseau ont été traités comme des indi. vidus supplémentaires, afin que la structure mise en évidence ne dépende que des échantillons du sous-écoulement.

Le premier axe factoriel explique $26,7 \%$ de la variance, le deuxième $24,9 \%$ et le troisième $19.9 \%$ soit $51,6 \%$ pour le plan principal F1-F2 et $71,5 \%$ pour l'hyperplan F1-F2-F3.

Ce pourcentage de variance expliquée est relativement faible, ce qui signifie que d'autres éléments interviennent, probablement, dans la discrimination des échantillons et. notamment, l'hétérogénéité spatiale, comme l'a laissé entrevoir l'analyse sommaire du tableau.

Dans l'espace des individus (plan 1-2, fig. 5) l'analyse révèle l'existence d'un cycle saisonnier ; en effet, les centres de gravité des différents groupes (T1, T2,.. T7), définis par la variable qualitative temps, se distribuent selon leur chronologie et les individus de septembre 1986 (T7) tendent à rejoindre ceux de septembre 1985 (T1). Deux facteurs déterminent ce cycle.

L'axe 1 oppose les individus de septembre et novembre à ceux de février, mars, mai et juillet : l'axe 2 oppose surtout ceux de juillet et septembre 1986 à ceux de novembre 1985 et février 1986.

$\mathrm{Si}$ l'on suit le déroulement de ce cycle, on constate une évolution assez rapide de septembre 1985 à février 1986, suivie d'un hiatus très net entre février et mars avec un regroupement des échantillons de mars, mai et juillet. Cette évolution suit les variations du chimisme du ruisseau puisque les individus de ce dernier (R1 à $R 7$ ) se placent toujours à proximité des centres de gravité (T1 à T7) des individus du sous-écoulement.

Les groupes d'échantillons mensuels sont bien définis puisque le pourcentage des individus bien classés dans ces groupes est de $92,5 \%$; seuls les groupes de mars et mai sont moins bien définis.

Dans l'espace des variables (fig. 6), l'axe 1 caractérise les eaux riches en sulfates et en magnésium qui s'opposent aux eaux pauvres en ces ions. L'axe 2 oppose les eaux riches en bicarbonates et celles relativement riches en $\mathrm{SiO}_{2}$ et $\mathrm{NO}_{3}$.

Par rapport à la variable implicite temps, le facteur 1 oppose les eaux d'étiage, riche's en $\mathrm{SO}_{4}$ et $\mathrm{Mg}$, aux hautes eaux. En septembre 1985, ces variables présentent respectivement des valeurs moyennes de 46,5 et $6,25 \mathrm{mg} / \mathrm{I}$, alors qu'en mars 1986 elles n'atteignent que 14,0 et $4,45 \mathrm{mg} / \mathrm{l}$ (tableau I). La spécificité des eaux d'étiage est due aux apports provenant du bassin versant du ruisseau de Plamicou dont on a vu qu'il est constitué par des terrains gypsifères riches en sulfates et magnésium.

Le facteur 2 indique l'existence de deux types de hautes eaux. Les premières marquées par de plus fortes teneurs en bicarbonates et en chlorures apparaissent en février 1986: elles traduisent la forte participation du système du Baget aux écoulements avec notamment des apports provenant de la zone de subsurface (Bakalowicz 1979). Les deuxièmes, qui se manifestent en juillet et septembre 1986 , sont caractérisées par leur enrichissement en $\mathrm{NO}_{3}$ et $\mathrm{SiO}_{2}$ dont l'origine est beaucoup moins évidente.

S'il est clair que les caractéristiques du sousécoulement suivent très précisément celles du cours subaérien au cours de l'évolution saisonnière, ce résultat n'implique pas pour autant une relation de causalité. Ainsi, les teneurs en $\mathrm{SO}_{4}$ sont toujours plus faibles dans le sous-écoulement que dans le ruisseau; ce résultat implique que l'eau du sousécoulement séjourne suffisamment longtemps dans les sédiments soit pour se mélanger avec une eat plus pauvre en $\mathrm{SO}_{4}$ (eau du karst), soit pour subir des réactions chimiques faisant disparaître $\mathrm{SO}_{4}$ (réduction). L'enrichissement observé en $\mathrm{NO}_{3}$ et $\mathrm{SiO}_{2}$ dans le sous-écoulement par rapport aux eaux de surface résulterait lui aussi d'un temps de séjour beaucoup plus long dans les sédiments; il est donc très probable que cet enrichissement observé au printemps soit lié non pas au ruissellement de surface sur le système karstique du Baget, mais plutôt à des phénomènes chimiques en aval de las Hountas. Les teneurs plus fortes en $\mathrm{HCO}_{3}$ et $\mathrm{CO}_{2}$ du sousécoulement rendent compte de l'absence d'échanges gazeux avec l'atmosphère, donc d'un écoulement souterrain.

Si la chimie des eaux du sous-écoulement ne peut donc être directement rattachée à celle des eaux superficielles situées à leur verticale, la similitude des profils géochimiques de ces deux types d'eau et leur évolution saisonnière synchrone traduisent cependant une dépendance certaine.

Ces données conduisent à proposer le modèle hydrologique suivant. Le sous-écoulement est essentiellement alimenté par une eau souterraine 


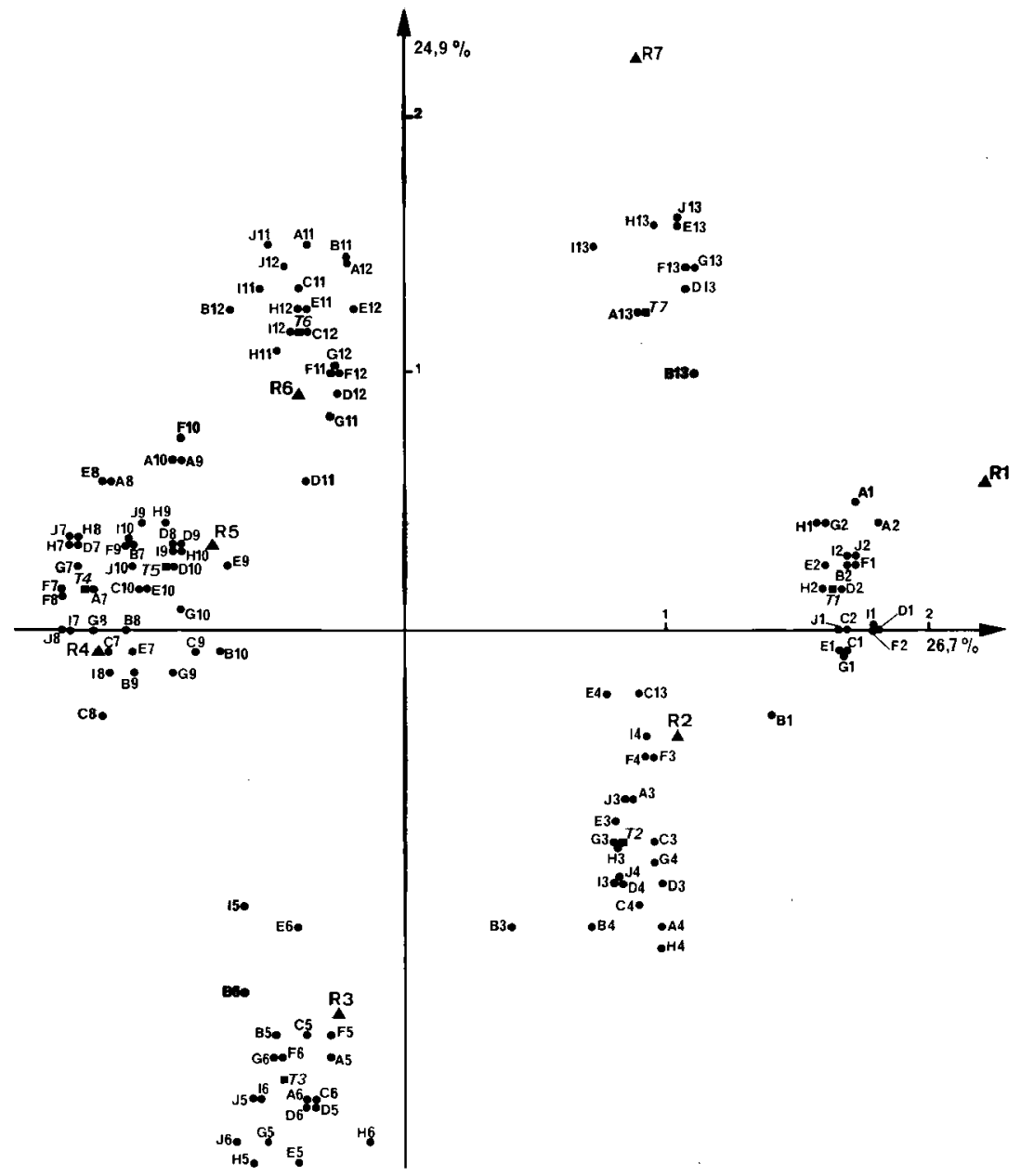

Fig. 5. Analyse factorielle discriminante en fonction du temps (espace des échantillons). La position des échantillons du cours superficiel est figurée par des triangles $\left(R_{1}, R_{2}, \ldots R_{7}\right)$ et celle des échantillons du sous-écoulement par des cercles. Le centre de gravité correspondant à chacune des périodes étudiées est représenté par un carré $\left(T_{1}, T_{2}, \ldots T_{7}\right)$. 


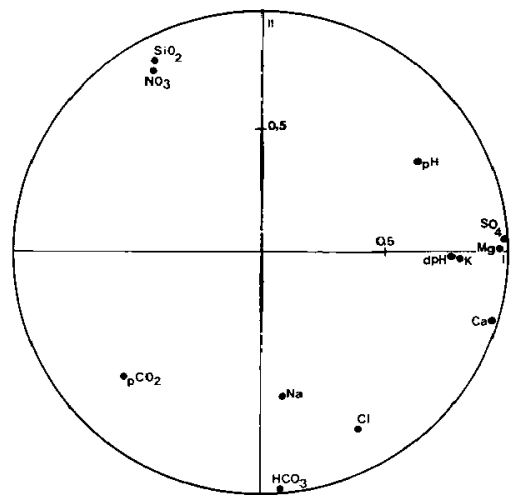

Fig. 6. Analyse factorielle discriminante en fonction du temps (espace des variables).

provenant de la nappe alluviale à $\mathrm{pCO}_{2}$ et à minéralisation plus élevées que celles de l'eau de surface. Cette eau souterraine reçoit de l'eau de surface comme l'indiquent notamment les apports sulfatés du ruisseau de Plamicou; mais cette alimentation est nécessai rement éloignée du site étudié, puisque les caractères d'eau souterraine, à cet endroit, ne sont pas altérés. La nappe alluviale serait alimentée à la fois directement par le karst et par le ruisseau de Lachein, en aval de Las Hountas, où des pertes sont connues.

\subsection{Variations du chimisme dans l'espace}

Dans cette analyse, la variable qualitative rend compte des différents secteurs définis sur le terrain. Les modalités suivantes ont été retenues: 1 pour le secteur $A, 2$ pour le secteur $B, \ldots 10$ pour le secteur $J$.

Le premier axe explique $52,2 \%$ de la variance, le deuxième $23.8 \%$, le troisième $9.7 \%$, soit, en pourcentage cumulé, 76,0\% pour le plan F1-F2 et 85,7 $\%$ pour l'hyperplan F1-F2-F3. La structure dans l'espace est par conséquent beaucoup plus forte que celle dans le temps.

Dans l'espace des individus (plan 1-2, fig. 7), les résultats permettent de séparer selon $\mathrm{F} 1$ les eaux du secteur B de celles de tous les autres secteurs; D apparaît intermédiaire. L'axe 2 oppose les eaux des secteurs $E$ et $G$ à celles des secteurs $A, J, H$ et, à un degré moindre, $F$; les secteurs C, I, D sont alors intermédiaires.

Le pourcentage des échantillons bien classés dans leur secteur est faible $(41,5 \%)$. Cela tient à la forme géométrique des secteurs, définie arbitrairement.

Dans l'espace des variables (fig. 8), l'axe 1 est caractérisé dans sa partie positive par un enrichissement en $\mathrm{HCO}_{3}$, $\mathrm{Ca}$ ainsi que par des $\mathrm{pCO}_{2}$ élevées et, dans sa partie négative, par de fortes teneurs en sulfates. On sait que le secteur $B$ constitue une aire réductrice comme en témoignent ses faibles teneurs en $\mathrm{O}_{2}$ dissous (Rouch 1988). Par conséquent, l'axe 1 caractérise un chimisme lié à l'oxygène, le pôle figuré par le secteur B rendant compte d'une évo. lution chimique par réduction dans les sédiments, donc, de l'absence de relations avec un milieu oxy. géné comme l'est le ruisseau.

La partie négative de l'axe 2 est caractérisée par un faible enrichissement en nitrates, un $\mathrm{pH}$ plus élevé et un dpH positif qui traduit une faible sursaturation (secteurs A, J, H, F). La partie positive de cet axe est définie par un enrichissement en $\mathrm{SiO}_{2}$ et Na (secteurs E, G).

La projection des échantillons du ruisseau à proximité des secteurs $\mathrm{A}, \mathrm{J}, \mathrm{H}$ et $\mathrm{F}$ conduit à penser que ce sont les secteurs dans lesquels le temps de séjour de l'eau dans le milieu n'est pas suffisamment long pour faire apparaître un accroissement notable des teneurs en $\mathrm{SiO}_{2}$ et $\mathrm{Na}$. Le fait que les $\mathrm{pCO}_{2}$ de ces secteurs soient un peu plus faibles que pour les autres traduit un mélange d'eau de surface avec l'eau souterraine, comme le confirment les dpH un peu plus forts ; mais cet apport d'eau du ruisseau reste très modeste et l'essentiel de l'eau du sousécoulement des secteurs $\mathrm{A}, \mathrm{J}, \mathrm{H}$ et $\mathrm{F}$ est d'origine souterraine. A l'opposé, les secteurs E et G traduisent des temps de séjour plus longs au contact de sédiments silicatés.

Ainsì, les eaux du sous-écoulement présentent une structure spatiale des faciès chimiques correspondant à trois types de milieu :

- les zones où le chimisme est voisin de celui de l'eau de surface, ce qui traduit un asport d'eau de surface modéré et un temps de séjour court dans un milieu à forte perméabilité : ce sont les secteurs $A$, J, H, F, à l'amont des bancs de graviers. Ces secteurs 
12

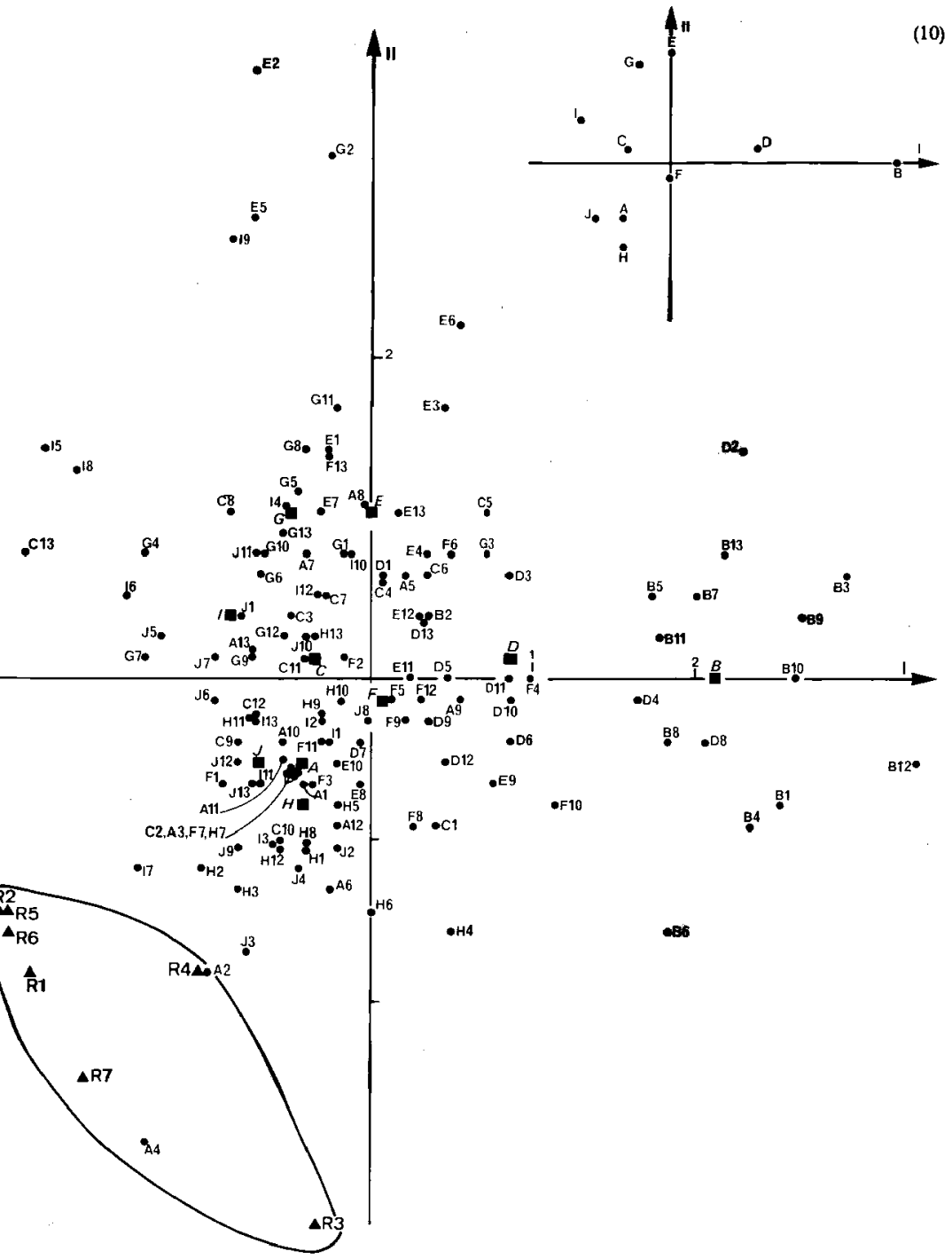

Fig. 7. Analyse factorielle discriminante en fonction de l'espace (espace des échantillons). La position des échantillons du cours superficiel est figurée par des triangles $\left(\mathbf{R}_{1}, \mathbf{R}_{2}, \ldots \mathbf{R}_{7}\right)$ et celle des échantillons du sous-écoulement par des cercles. Le centre de gravité correspondant à chacun des secteurs du site est représenté par un carré $(A, B, C, \ldots J)$. Dans le schéma en haut et à droite de la figure, seule la position des centres de gravité est rappelée. 


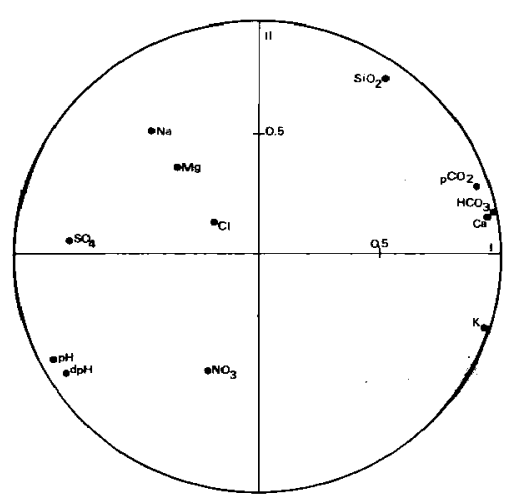

Fig. 8. Analyse factorielle discriminante en fonction de l'espace (espace des variables).

présentent la plus forte porosité et les teneurs les plus élevées en $\mathrm{O}_{2}$ dissous (Rouch 1988).

- la zone ou le chimisme présente une nette soussaturation en $\mathrm{HCO}_{3}$, de fortes teneurs en $\mathrm{CO}_{2}$ et en silice, et une réduction des sulfates, ce qui implique l'absence d'apport d'eau de surface et un temps de séjour long dans un milieu confiné à faible perméabilité. Ce secteur (B) est effectivement caractérisé par de très faibles valeurs en $\mathrm{O}_{2}$ dissous et une porosité inférieure aux zones précédentes.

- une zone où le chimisme est caractérisé par un accroissement net des teneurs en $\mathrm{SiO}_{2}$ et en $\mathrm{Na}$ (secteurs $E$ et $G$ du chenal), ce qui est la preuve de l'absence d'apports notables d'eau de surface et d'un temps de séjour assez long dans un milieu à perméabilité faible, mais sans confinement. Les porosités y sont faibles et les teneurs en $\mathrm{O}_{2}$ dissous intermédiaires entre les 2 cas précédents.

\subsection{Cartographie de la structure chimique du sous-écoulement}

L'analyse précédente montre que de nombreux échantillons sont mal classés et que certains secteurs ( $C, D, I)$ sont hétérogènes. Afin de préciser la structure chimique du site étudié et d'en proposer une cartographie, une nouvelle AFD a été réalisée où n'ont été retenues que les trois modalités mises en évidence dans l'analyse précédente : 1 pour la zone réductrice (type $=$ secteur $\mathrm{B}$ ), 2 pour la zone à forte perméabilité (types $=$ secteurs $\mathrm{A}, \mathrm{J}, \mathrm{H}, \mathrm{F}$ à l'amont des bancs de graviers), 3 pour la zone à faible perméabilité (types = secteurs E et $\mathrm{G}$ du chenal).

Les individus des zones $C, D, I$ qui paraissaient intermédiaires ont été traités en individus supplémentaires. Cette nouvelle analyse fournit des résultats semblables à la précédente. Mais le fait de n'avoir retenu que 3 modalités renforce la détermination de la structure : $65,9 \%$ de l'inertie sont expliqués par l'axe 1 et $34,1 \%$ par l'axe 2 , soit, en pourcentage cumulé $100 \%$. Ce résultat prouve, a posteriori, le bien fondé du choix réalisé et montre également que, dans l'AFD précédente, il était inutile de chercher une interprétation pour l'axe $3.84,6 \%$ des échantillons sont désormais bien classés.

Grâce, d'une part, au tableau des coordonnées des individus désignant l'affectation de chacun au groupe le plus proche et, d'autre part, au tableau des coordonnées des individus supplémentaires donnant l'affectation de ceux.ci aux trois groupes, il est possible de proposer une cartographie des faciès du chimisme des eaux du sous-écoulement ( $f$ ig. 9).

La distribution des échantillons sur le site souligne la spécificité, sur le plan chimique, des trois grands ensembles reconnus. Elle permet, également, de comprendre la diversité de certains secteurs qui, dans l'analyse précédente, présentaient des caractères intermédiaires : leur hétérogénéité est due à leur position topographique. Le secteur D est influencé par la zone réductrice, le chenal et les bancs de graviers, les secteurs $\mathbf{C}$ et I par le chenal et les bancs de graviers. Ainsi, les ensembles physiographiques définis par les écoulements de surface peuvent être différenciés aussi bien sur le plan physique que sur le plan géochimique.

\section{Conclusions}

La plupart des études écologiques con sacrées au sous-écoulement des cours d'eau ont conduit leurs auteurs à souligner les fortes analogies qui, sur le plan chimique, existent entre le cours subaérien et son sous-écoulement (Angelier 1953, Schwoerbel 1961, Ruttner-Kolisko 1961, Tilzer 1968, Husmann 1971, Williams \& Hynes 1974, Danielopol 1976, 


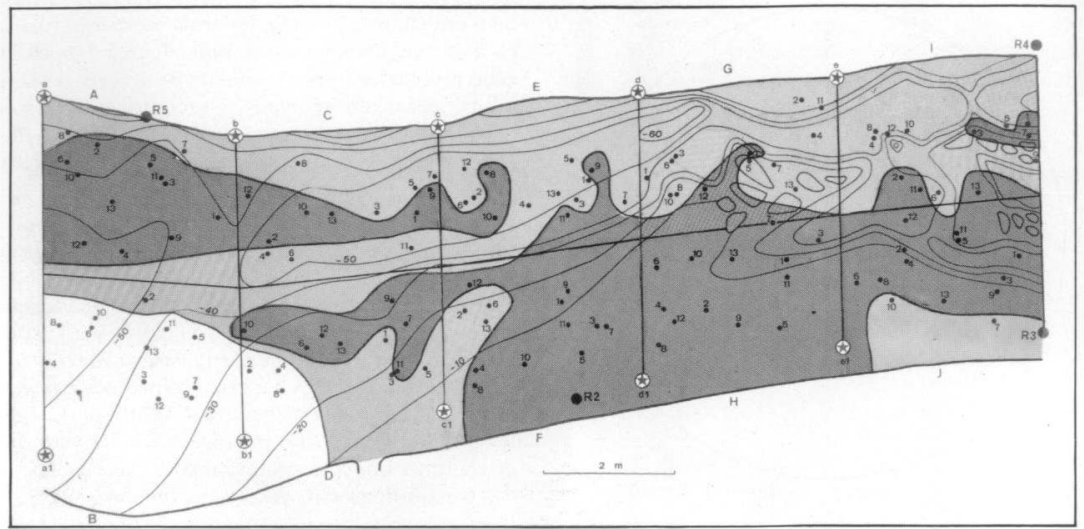

Fig. 9. Cartographic des facies géochimiques du sous-écoulement : en blanc, la zone réductrice, appauvrie en sulfates et enrichie en $\mathrm{CO}_{2}$ et en bicarbonates; en gris clair, la zone du chenal enrichie en silice et en sodium; en gris foncé, les bancs de graviers plus pauvres en $\mathrm{CO}_{2}$ et bicarbonates.

1983, Ferrarese \& Sambugar 1976, Braioni et al. 1980, Reygrobellet et al. 1981, Bretschko 1981, Hynes 1983, Dole 1983, Mestrov et al. 1983, Whitman \& Clark 1984, Standford \& Ward 1988).

C'est également vrai pour le ruisseau de Lachein et son sous-écoulement. Dans son cas, cette analogie paraît renforcée par le fait que les caractéristiques chimiques du sous-écoulement et du cours subaérien évoluent de façon synchrone au fil des saisons.

Toutefois, le chimisme du sous-écoulement du ruisseau de Lachein n'est pas sous le contrôle direct de celui de l'écoulement de surface auquel il est associé. En effet, certains caractères géochimiques (la $\mathrm{pCO}_{2}$, le $\mathrm{dpH}$ et les variables associées $\mathrm{Ca}$ et $\mathrm{HCO}_{3}$ ) prouvent l'indépendance relative de ces deux types d'eau. Alors que l'eau du ruisseau perd, lors de son parcours aérien, une partie de son $\mathrm{CO}_{2}$, $\mathrm{HCO}_{3}$ et $\mathrm{Ca}$ et tend vers un déséquilibre calcocarbonique bien marqué (dpH nettement positif), l'eau du sous-écoulement conserve des caractères très nets d'eau souterraine. L'explication d'un tel phénomène réside dans la dualité suivante : les eaux de surface et du sous-écoulement appartiennent à un même ensemble hydrologique, d'où leurs ressemblances ; toutefois, ces eaux subissent une évolution différente selon le milieu dans lequel elles circulent, les unes en surface, les autres en profondeur. Les échanges entre ces deux flux ne sont jamais suffisamment importants pour changer fondamentalement le chimisme de chacun des écoulements.

Dans l'aire étudiée, le chimisme de l'eau rend particulièrement bien compte de l'hétérogénéité spatiale du sous-écoulement. En effet, il existe une structure des faciès chimiques de ses eaux qui est directement liée à la physiographie des sédiments. Dans les bancs de graviers où la perméabilité est élevée, la sursaturation calco-carbonique est plus importante que dans les zones à plus faible perméabilité, le chenal et la zone réductrice. Ces dernières sont marquées par un net enrichissement en $\mathrm{SiO}_{2}$ dû à un temps de séjour des eaux plus long qui permet une dissolution des sédiments silicatés. En outre, dans la zone réduct rice où le confinement est important, les sulfates sont partiellement réduits.

La car tographie de cette structure géochimique est tout à fait comparable à celle obtenue avec d'autres données (voir Rouch 1988), granulométrie et 
porosité, $\mathrm{O}_{2}$ dissous, densités des Harpacticides, distribution du pourcentage des Harpacticides hypogés, distrit?:ition des espèces de Crustacés stygobies.

Ainsi, la partition des habitats provoquée par les écoulements de surface se traduit par une ségrégation du milieu tant physique que chimique et biologique.

Les résultats obtenus prouvent que l'hydrochimie est un outil tout à fait adapté pour décrire les écoulements souterrains et la nature de leur hétérogénéité comme cela a été également démontré pour les aquifères karstiques (Bakalowicz 1979). Le chimisme du sous-écoulement, comme celui du ruisseau de Lachein, est la résultante de phénomènes se produisant à des échelles très différentes : à l'échelle du bassin versant (chimisme calco-carbonique, eaux enrichies en $\mathrm{Cl}$ en début de recharge), à l'échelle des sous-bassins (apports sulfatés du ruisseau de Plamicou), à l'échelle du site (distinction entre le ruisseau et le sous-écoulement), à l'échelle métrique et décimétrique (définition de zones à perméabilité très différente dans le sous-écoulement). Ainsi, il apparaît très clairement que la mise en évidence d'une structure spatiale et temporelle du chimisme des eaux et la recherche de ses causes doivent nécessairement s'appuyer sur une connaissance suffisante de l'ensemble du système hydrologique.

Au point de vue technique, ce travail permet également d'apporter des informations sur la méthode des sondages tubés. On sait que, outre sa facilité de mise en œuvre qui permet d'effectuer un grand nombre d'observations sans al térer significativement le milieu, elle fournit la possibilité d'associer échantillonnages biologique et chimique. La question s'est posée de savoir quelle était la représentativité de tels échantillons (Exner \& Davies 1979). Dès 1976, Danielopol indiquait que les animaux présents dans les 10 premiers litres pompés étaient représentatifs de la communauté située à proximité de la crépine. Dans le sous-écoulement du ruisseau de Lachein, compte tenu d'une porosité moyenne des sédiments de $30 \%$ (Rouch 1988), et d'une hauteur de la crépine de $15 \mathrm{~cm}$, le rayon d'influence d'un pompage correspondant à $10 \mathrm{l}$ peut être estimé à $26 \mathrm{~cm}$ autour du tube. La distribution des échantillons sur le site en fonction de leurs caractéristiques géochimiques ainsi que les limites qui en découlent, en accord avec l'organisation spatiale du milieu, confirment cette estimation. Dans le plan horizonta], la méthode des sondages tubés fournit donc des échantillons biologiques et chimiques représentatifs de l'échelle décimétrique.

\section{Remerciements}

Nous remercions bien vivement Madame A. Descouens sans l'aide de cui ce travail n'aurait pu être mené à bien, que ce soit pour le recueil des données sur le terrain ou pour la réalisation de l'iconographie de cette note, ainsi que Madame J. Daffis qui a effectué les analyses chimiques qui sont le fondement de cet article. Nous nemercions également Madame F. Boineau qui a saisi le manuscrit.

\section{Travaux chtés}

Angelier (E.). 1953. - Recherches écologiques et biogéographiques sur la faune des sables submergés. Arch. Zool. exp. gén., $90: 37-161$.

Bakalowicz (M.). 1979. - Contribution de la géochimie des eaux à la connaissance de l'aquifère karstique et de la karstification. Thèse Doct. Sciences, Univ. Paris-6: $269 \mathrm{p}$.

Bakalowicz (M.) \& Aminot (A.). 1974. - Géochimie des eaux d'aquifëres karstiques. III. Premiers résultats obtenus sur le système karstique du Baget durant le cycle hydrologique 1973. Arn Spéléol, 29 : 484-493.

Bou (C.) \& Rouch (R.). 1967, - Un nouveau champ de recherches sur la faune aquatique souterraine. C.R. Acad. Sc. Paris, 265 : 369-370.

Braioni (M.G.), Duzzin (B.), Centurioni (M.C.), Penna (G.), Negroni (L) \& Campaioli (S.). 1980. - L'ambiente interstiziale iporreico del fiume Brenta e la sua fauna. Boll. Mus. Civ. St. Nat. Verona, $7: 235.274$.

Bretschko (G.). 1981. - Vertical distribution of zoobenthos in an alpine brook of the Ritrodat-Lunz study area. Verh. Intermat. Verein. Limnol, $21: 873-876$.

Danielopol (D.). 1976. - The distribution of the fauna in the interstitial habitats of riverine sediments of the Danube and Piesting (Austria). Int. J. Speleol., $8: 23-51$.

Danielopol (D.). 1983. - Der Einfluss organischer Verschmutaung auf das Grundwasser-Ökosystem der Donau im Raum Wien und Niederösterreich. Bundesministerium f. Gesundheit u. Umweltschutz, Wien, Forschumgsberichte, 5: 5-160.

Dole (M.J.) 1983. - Le domaine aquatique souterrain de la plaine al luvia le du Rhône à l'est de Lyon ; écologie des niveaux supérieurs de la nappe. Thèse de $3^{\text {e }}$ cycle, Univ. Claude Bernard. Lyon I : $168 \mathrm{p}$.

Exner (K.K.) \& Davies (R.W.). 1979. - Comments on the use of a stancipipe corer in fluvial gravels. Freshw. Biol., 9: 77-78.

Ferrarese (V.) \& Sambugar (B.). 1976. - Ricerche sulla fauna interstiziale iporreica dell'Adige in relazione allo stato di inquinamento del fiume. Riv. Idrobiol., 15 : 47-127.

Husmann (S.). 1971. - Eine neue Methode zur Entnahme von Interst itialwasser aus subaquat ischen Lockergesteinen. Arch. Hydrobiol., 68 : 519-527.

Hynes (H.B.N.). 1983. - Ground water and stream ecology. Hydro biologia, 100: 93-99.

Mest rov (M.), Stilinovic (B.), Habdija (I.), Lattinger (R.), Maloseja (Z.), Kerovec (M.) \& Cicin-Sain (L). 1983. - The ecological characteristics of interstitial undergrom the water of the river Sava. Acia Biologica, $9: 5-53$. 
Reygrobellet (J.L.), Mathicu (J.), Ginet (R.) \& Gibert (J.). 1981 . Structure et fonctionnement des écosystèmes du Haut-Rhône français; VIII : Hydrologie de deux stations phréatiques dont l'eau alimente les bras morts. Int. J. Speleol., 11 : 129-139.

Rouch (R.). 1988. - Sur la répartition spatiale des Crustacés dans le sous-écoulement d'un ruisseau des Pyrénées. Annls. Limnol., 24 : 213-234.

Ruttner-Kolisko (A.). 196 I. - Biotop und Biozönose des Sandufers einiger österreichischer Flüsse. Vehr. Intemat. Verein. Limnol, 14 : 362-368.

Schwoerbel (J.) I961. - Uber die Lebensbedingungen und dic Besiedlung des hyporheischen Lebensraumes. Arch. Hydrobiol., suppl., $25: 182-214$
Standford (J.A.) \& Ward (J.V.). 1988. - The hyporheic habitat of river ecosystems. Nature, 335: 64-66.

Tilzer (M.). 1968. - Zur Ökologie und Besiedlung des hochalpinen hyporheischen Interstitials im Arlberggebiet (Österreich). Arch. Hydrobiol, 65 : 253-308.

Whitman (R.L.) \& Clark (W.J.). 1982. - Availability of dissolved oxygen in interstitial waters of a sandy creek. Hydrobiologia, 92: 651-658.

Williams (D.D.) \& Hynes (H.B.N.) 1974 - The occurence of benthos deep in the substratum of a stream. Freshwat. Biol., 4 : 233-256. 